\title{
Spreading of Messages in Random Graphs
}

\author{
Ching-Lueh Chang ${ }^{1} \quad$ Yuh-Dauh Lyuu ${ }^{2}$
}

\author{
${ }^{1}$ Department of Computer Science and Information Engineering \\ National Taiwan University \\ Taipei, Taiwan \\ Email: d95007@csie.ntu.edu.tw \\ 2 Department of Computer Science and Information Engineering \\ National Taiwan University \\ Taipei, Taiwan \\ Email: lyuu@csie.ntu.edu.tw
}

\begin{abstract}
Chang and Lyuu [Chang and Lyuu, 2008] study the spreading of a message in an Erdős-Rényi random graph $G(n, p)$ starting from a set of vertices that are convinced of the message initially. In their strictmajority scenario, whenever more than half of the neighbors of a vertex $v$ are convinced of a message, $v$ itself also becomes convinced. The spreading proceeds asynchronously until no more vertices can be convinced. Following Chang and Lyuu [Chang and Lyuu, 2008], we derive lower bounds on the minimum number min-seed $(n, p)$ of vertices that need to be convinced initially so that all vertices will be convinced at the end. Our main results are that $\min -\operatorname{seed}(n, p)=$ $\Omega\left(\min \left\{n, p^{2} n^{2}\right\}\right)$ and $\min -\operatorname{seed}(n, p)=\Omega\left(n^{2 / 3}\right)$ hold with high probability. We also consider the case of random seeds. For any sufficiently large constant $d>0$ and any $s \leq n /(d \ln n)$, we show that if one picks the set of seeds uniformly at random from the family of all $s$-sized sets, then with high probability, not all vertices will be convinced at the end.
\end{abstract}

\section{Introduction}

Chang and Lyuu [Chang and Lyuu, 2008] study the spreading of messages in undirected graphs under the assumption that each vertex is convinced of a message when more than half of its neighboring vertices are convinced. In their setting, a message initially convinces a set of vertices called the seeds. Then the spreading process proceeds asynchronously and a vertex is convinced of the message when more than half of its neighboring vertices are convinced. The spreading ends when no more vertices can be convinced.

This paper focuses on the case where the underlying graph in which messages spread is the ErdösRényi random graph $G(n, p), n \in \mathbb{N}, p \in[0,1]$ [Bollobás, 2001]. Denote by $\min -\operatorname{seed}(n, p)$ the minimum number of seeds needed to convince all vertices of $G(n, p)$ at the end. Chang and Lyuu [Chang and Lyuu, 2008] show that $\min -\operatorname{seed}(n, p)=$

Research supported in part by NSC grant 96-2213-E-002-024.

Copyright (C)2009, Australian Computer Society, Inc. This paper appeared at the Fifteenth Computing: The Australasian Theory Symposium (CATS 2009), Wellington, New Zealand. Conferences in Research and Practice in Information Technology (CRPIT), Vol. 94, Rod Downey and Prabhu Manyem, Ed Reproduction for academic, not-for profit purposes permitted provided this text is included.
$\Omega(\min \{n, 1 / p\})$ holds with high probability over $G(n, p), n \in \mathbb{N}, p \in(0,1]$. However, their $\Omega(\min \{n, 1 / p\})$ lower bound weakens as $p$ increases. In this paper, we prove an $\Omega\left(\min \left\{n, p^{2} n^{2}\right\}\right)$ lower bound on $\min -\operatorname{seed}(n, p)$ that holds with high probability over $G(n, p)$. This complements the previous $\Omega(\min \{n, 1 / p\})$ lower bound in that the new bound grows as $p$ increases. Combining these two lower bounds, we prove that $\min -\operatorname{seed}(n, p)=\Omega\left(n^{2 / 3}\right)$ holds with high probability over $G(n, p)$, thereby establishing a lower bound on $\min -\operatorname{seed}(n, p)$ that is independent of $p$. In addition, the proof of our $\Omega\left(\min \left\{n, p^{2} n^{2}\right\}\right)$ lower bound is extended to deal with the convincing of a $\delta$ fraction of vertices for any $\delta \in(0,1]$.

We also consider the case of random seeds. For any sufficiently large constant $d>0$ and any $s \leq$ $n /(d \ln n)$, we show that if one picks the set of seeds uniformly at random from the family of all $s$-sized sets, then with high probability, some vertices will never be convinced.

Prior to our paper, Watts [Watts, 2002], Gleeson and Cahalane [Gleeson and Cahalane, 2007] and Chang and Lyuu [Chang and Lyuu, 2008] also consider the more general case where each vertex $v$ is assigned a threshold $\alpha(v)$ and is convinced of the message when at least $\alpha(v)$ of $v$ 's neighbors are convinced. However, unlike the work of Chang and Lyuu [Chang and Lyuu, 2008] and the current paper, Watts [Watts, 2002] and Gleeson and Cahalane [Gleeson and Cahalane, 2007] do not prove (lower or upper) bounds on the minimum number of vertices needed to convince all or a large fraction of vertices in a graph.

Analogous to the convincing of vertices in our setup, neurons in neural networks often adopt threshold activation functions to determine whether to fire [Haykin, 1998]. However, our spreading process has the objective of convincing all vertices, whereas neural networks do not aim at making all neurons fire.

Schelling [Schelling, 1973] studies the general phenomenon of binary choices with externalities, wherein the involved individuals may adopt any particular mechanism to make binary choices with the aid of available information from others. Sensibly, such a setting is general enough to model a broad class of scenarios like those in this paper and Watts' paper, among others (see, e.g., those mentioned by Watts). But our results are independent from those of Schelling.

There are also the related issues of rumor spreading [Karp et al., 2000, Doerr et al., 2008], broadcasting, accumulation and gossiping in interconnection networks [Hedetniemi et al., 1988, Hromkovic et al., 1996], but our setting is different from these in that, under our setup, a vertex may not believe a message 
even if some of its neighboring vertices do.

Our lower bounds can also be viewed from the perspective of trust propagation [Grandison and Sloman, 2000, Artz and Gil, 2007] in that we provide rigorously provable limitations on propagating the individuals' trust in messages.

This paper is organized as follows. Section 2 defines our model for the spreading of messages and introduces notations to be used later. The model is a special case of that of Watts [Watts, 2002] and Chang and Lyuu [Chang and Lyuu, 2008]. Section 3 briefly describes the known results needed in later sections. Section 4 presents our lower bounds on $\min -\operatorname{seed}(n, p)$. Section 5 extends our treatment to the case of random seeds. Section 6 concludes the paper.

\section{Definitions and notations}

We follow Chang and Lyuu's [Chang and Lyuu, 2008] setup on the spreading of messages. The underlying network in which messages spread is the ErdösRényi random graph $G(n, p)$, which is a graph with vertices $1, \ldots, n$ where each of the possible $\left(\begin{array}{l}n \\ 2\end{array}\right)$ edges appears independently with probability $p$ [Bollobás, 2001]. The spreading of a message begins from a set of vertices, called the seeds, which are convinced of the message initially. Subsequently, each vertex becomes convinced after more than half of its neighboring vertices are convinced. The spreading proceeds asynchronously until no more vertices can be convinced.

We follow the notations of Chang and Lyuu [Chang and Lyuu, 2008]. In particular, we write $n] \equiv\{1, \ldots, n\}$ and $N(v)$ for the set of $v$ 's neighbors in $G(n, p), v \in[n]$. We denote by $c(S)$ the set of vertices of $G(n, p)$ which are convinced at the end, given that $S \subseteq[n]$ is the set of seeds. Clearly, $S \subseteq c(S)$ for every $S \subseteq[n]$. As a slight simplification of Chang and Lyuu's notations, we write $\min -\operatorname{seed}(n, p)$ for the minimum number of seeds needed to convince all vertices of $G(n, p)$. Note that $N(v), c(S)$ and $\min -\operatorname{seed}(n, p)$ are all random variables as $G(n, p)$ is a random graph.

\section{Preliminaries}

Before establishing our lower bounds on $\min$-seed $(n, p)$, we state several known results that will be useful later. The following facts are the lower and upper tails of the Chernoff bound [Chernoff, 1952, Motwani and Raghaven, 1995].

Fact 1. Let $X_{1}, \ldots X_{m}$ be independent Bernoulli trials with $\operatorname{Pr}\left[X_{i}=1\right]=p, 1 \leq i \leq m$, and write $\mu=E\left[\sum_{i=1}^{m} X_{i}\right]$. Then for any $\delta \in(0,1)$,

$$
\operatorname{Pr}\left[\sum_{i=1}^{m} X_{i}<(1-\delta) \mu\right]<\exp \left(-\frac{\delta^{2} \mu}{2}\right) .
$$

Fact 2. Let $X_{1}, \ldots X_{m}$ be independent Bernoulli trials with $\operatorname{Pr}\left[X_{i}=1\right]=p, 1 \leq i \leq m$, and write $\mu=E\left[\sum_{i=1}^{m} X_{i}\right]$. Then for any $\delta>0$,

$$
\operatorname{Pr}\left[\sum_{i=1}^{m} X_{i}>(1+\delta) \mu\right]<\left(\frac{\exp (\delta)}{(1+\delta)^{1+\delta}}\right)^{\mu} .
$$

We will also use the following well-known fact.

Fact 3. ([Motwani and Raghaven, 1995]) For any positive integers $s \leq n$,

$$
\left(\begin{array}{l}
n \\
s
\end{array}\right) \leq\left(\frac{e n}{s}\right)^{s}
$$

The following theorem is due to Chang and Lyuu [Chang and Lyuu, 2008].

Theorem 4. ([Chang and Lyuu, 2008]) Let $n$ be a positive integer and $p \in(0,1]$. With probability $1-$ $o(1)$,

$$
\min -\operatorname{seed}(n, p)=\Omega\left(\min \left\{n, \frac{1}{p}\right\}\right) .
$$

\section{Spreading of messages in random graphs}

In this section, we derive lower bounds on $\min -\operatorname{seed}(n, p)$. For this purpose, we introduce several lemmas below.

Lemma 5. Let $p \in[0,1], n, s$ be positive integers with $s \leq n / 100$ and $S \subsetneq[n]$ have cardinality s. If $|c(S) \backslash \bar{S}| \geq p n / 8$ and $|\hat{N}(v)|>p n / 2$ for all $v \in[n]$, then there exist distinct vertices $v_{1}, \ldots, v_{p n / 8} \in[n] \backslash S$ with $\left|N\left(v_{k}\right) \cap S\right|>$ pn $/ 8$ for $1 \leq k \leq p n / 8$.

Proof. Clearly, the elements of $c(S) \backslash S$ can be sequenced as $v_{1}, \ldots, v_{|c(S) \backslash S|}$ such that given $S$ as the set of seeds, the vertices in $c(S) \backslash S$ are convinced in that order. By definition,

$$
\left|N\left(v_{k}\right) \cap S\right|+\left|\left\{v_{1}, \ldots, v_{k-1}\right\}\right|>\frac{\left|N\left(v_{k}\right)\right|}{2},
$$

$1 \leq k \leq|c(S) \backslash S|$. As $|N(v)| \geq p n / 2$ for each $v \in[n]$, for $1 \leq k \leq p n / 8$,

$$
\left|N\left(v_{k}\right) \cap S\right|>\frac{\left|N\left(v_{k}\right)\right|}{2}-(k-1) \geq \frac{p n}{4}-\frac{p n}{8}=\frac{p n}{8} .
$$

Lemma 6. Let $p \in[0,1]$ and $n, s$ be positive integers with $s \leq n / 100$. For each $S \subsetneq[n]$ of size $s$,

$$
\begin{aligned}
& \operatorname{Pr}\left[\left(|c(S) \backslash S| \geq \frac{p n}{8}\right) \wedge\left(\forall v \in[n],|N(v)| \geq \frac{p n}{2}\right)\right] \\
\leq & \left(\begin{array}{c}
n-s \\
\frac{p n}{8}
\end{array}\right) \exp \left(-\frac{p^{2} n^{2}}{128} \ln \frac{n}{8 s}\right),
\end{aligned}
$$

where the probability is taken over the random graphs $G(n, p)$.

Proof. In the proof, all probabilities are taken over the random graphs $G(n, p)$. By Lemma 5 ,

$$
\operatorname{Pr}\left[\left(|c(S) \backslash S| \geq \frac{p n}{8}\right) \wedge\left(\forall v \in[n],|N(v)| \geq \frac{p n}{2}\right)\right]
$$

$\leq \operatorname{Pr}\left[\exists\right.$ distinct $v_{1}, \ldots, v_{p n / 8} \in[n] \backslash S$ such that

$$
\begin{aligned}
& \left.\forall k, 1 \leq k \leq \frac{p n}{8},\left|N\left(v_{k}\right) \cap S\right|>\frac{p n}{8}\right] \\
\leq \quad & \operatorname{Pr}\left[\forall k, 1 \leq k \leq \frac{p n}{8},\right.
\end{aligned}
$$

distinct $v_{1}, \ldots, v_{p n / 8} \in[n] \backslash S$

$$
\begin{aligned}
& \left.\left|N\left(v_{k}\right) \cap S\right|>\frac{p n}{8}\right] \\
\leq & \left(\begin{array}{c}
n-s \\
\frac{p n}{8}
\end{array}\right)_{\text {distinct } v_{1}, \ldots, v_{p n / 8} \in[n] \backslash S} \operatorname{Pr}\left[\forall k, 1 \leq k \leq \frac{p n}{8}\right. \\
& \left.\left|N\left(v_{k}\right) \cap S\right|>\frac{p n}{8}\right] .
\end{aligned}
$$

By the definition of Erdös-Rényi random graphs, the random variables $|N(v) \cap S|$ for $v \in[n] \backslash S$ are 
independently and identically distributed. Hence for any distinct $v_{1}, \ldots, v_{p n / 8} \in[n] \backslash S$ and any $u \in[n] \backslash S$,

$$
\begin{aligned}
& \operatorname{Pr}\left[\forall k, 1 \leq k \leq \frac{p n}{8},\left|N\left(v_{k}\right) \cap S\right|>\frac{p n}{8}\right] \\
= & \prod_{k=1}^{\frac{p n}{8}} \operatorname{Pr}\left[\left|N\left(v_{k}\right) \cap S\right|>\frac{p n}{8}\right] \\
= & \left(\operatorname{Pr}\left[|N(u) \cap S|>\frac{p n}{8}\right]\right)^{\frac{p n}{8}} .
\end{aligned}
$$

Fact 2 implies that for each $u \in[n] \backslash S$,

$$
\begin{aligned}
& \operatorname{Pr}\left[|N(u) \cap S|>\frac{p n}{8}\right] \\
= & \operatorname{Pr}\left[|N(u) \cap S|>\frac{n}{8 s} p s\right] \\
= & \operatorname{Pr}\left[|N(u) \cap S|>\frac{n}{8 s} E[|N(u) \cap S|]\right] \\
< & \left(\frac{\exp \left(\frac{n}{8 s}-1\right)}{\left(\frac{n}{8 s}\right)^{\frac{n}{8 s}}}\right)^{p s} \\
< & \exp \left(-\frac{p n}{16} \ln \frac{n}{8 s}\right),
\end{aligned}
$$

where the last inequality follows from $s \leq n / 100$ and straightforward calculations. This and Eqs. (1)-(2) complete the proof.

We now establish the following lower bound on $\min -\operatorname{seed}(n, p)$.

Theorem 7. Let $n$ be a positive integer and $p \in$ $[0,1]$. With probability $1-o(1)$,

$$
\min -\operatorname{seed}(n, p)=\Omega\left(\min \left\{n, p^{2} n^{2}\right\}\right) .
$$

Proof. In the sequel, all the probabilities are taken over the random graphs $G(n, p)$. For $p<32(\ln n) / n$, we have $p^{2} n^{2}=o(1 / p)$ and therefore the lower bound on min-seed $(n, p)$ follows from Theorem 4 .

Now assume $p \geq 32(\ln n) / n$ and let $s \leq n / 100$ be an arbitrary positive integer. We have

$$
\begin{aligned}
& \operatorname{Pr}[\min -\operatorname{seed}(n, p) \leq s] \\
= & \operatorname{Pr}[\exists S \subsetneq[n],|S|=s, c(S)=[n]] \\
\leq & \operatorname{Pr}\left[\exists S \subsetneq[n],|S|=s,|c(S) \backslash S| \geq \frac{p n}{8}\right] \\
\leq & \operatorname{Pr}\left[\exists v \in[n],|N(v)|<\frac{p n}{2}\right] \\
& +\operatorname{Pr}\left[\left(\exists S \subsetneq[n],|S|=s,|c(S) \backslash S| \geq \frac{p n}{8}\right)\right. \\
& \left.\wedge\left(\forall v \in[n],|N(v)| \geq \frac{p n}{2}\right)\right] \\
\leq & \sum_{v \in[n]} \operatorname{Pr}\left[|N(v)|<\frac{p n}{2}\right] \\
& +\sum_{S \subsetneq[n],|S|=s} \operatorname{Pr}\left[\left(|c(S) \backslash S| \geq \frac{p n}{8}\right)\right. \\
& \left.\wedge\left(\forall v \in[n],|N(v)| \geq \frac{p n}{2}\right)\right] \\
\leq & \sum_{v \in[n]} \operatorname{Pr}\left[|N(v)|<\frac{p n}{2}\right] \\
& +\left(\begin{array}{c}
n \\
s
\end{array}\right)\left(\begin{array}{c}
n-s \\
\frac{p n}{8}
\end{array}\right) \exp \left(-\frac{p^{2} n^{2}}{128} \ln \frac{n}{8 s}\right),
\end{aligned}
$$

where the last inequality follows from Lemma 6 .
Now Fact 1 implies that for $v \in[n]$,

$$
\begin{aligned}
& \operatorname{Pr}\left[|N(v)|<\frac{p n}{2}\right] \\
= & \operatorname{Pr}\left[|N(v)|<\frac{n}{2(n-1)} p(n-1)\right] \\
= & \operatorname{Pr}\left[|N(v)|<\frac{n}{2(n-1)} E[|N(v)|]\right] \\
< & \exp \left(-\frac{p n}{9}\right) .
\end{aligned}
$$

Combining Eqs. (3)-(4),

$$
\begin{aligned}
& \operatorname{Pr}[\min -\operatorname{seed}(n, p) \leq s] \\
< & n \exp \left(-\frac{p n}{9}\right)+\left(\begin{array}{c}
n \\
s
\end{array}\right)\left(\begin{array}{c}
n-s \\
\frac{p n}{8}
\end{array}\right) \exp \left(-\frac{p^{2} n^{2}}{128} \ln \frac{n}{8 s}\right) \\
\leq & n \exp \left(-\frac{p n}{9}\right)+\left(\frac{e n}{s}\right)^{s} n^{\frac{p n}{8}} \exp \left(-\frac{p^{2} n^{2}}{128} \ln \frac{n}{8 s}\right) \\
= & n \exp \left(-\frac{p n}{9}\right)+\exp \left(s+s \ln \frac{n}{s}+\frac{p n}{8} \ln n-\frac{p^{2} n^{2}}{128} \ln \frac{n}{8 s}\right) \\
< & o(1)+\exp \left(s+s \ln \frac{n}{s}-\frac{p^{2} n^{2}}{256} \ln \frac{n}{8 s}\right),
\end{aligned}
$$

where the second inequality uses Fact 3 to deduce $\left(\begin{array}{l}n \\ s\end{array}\right) \leq(e n / s)^{s}$ and the last inequality follows from $p \geq 32(\ln n) / n, s \leq n / 100$ and straightforward calculations.

By setting $s=\min \left\{n / 100, \epsilon p^{2} n^{2}\right\}$ for a sufficiently small constant $\epsilon>0$ in Eq. (5),

$$
\begin{aligned}
& \operatorname{Pr}[\min -\operatorname{seed}(n, p) \leq s] \\
\leq & o(1)+\exp \left(-\Omega\left(p^{2} n^{2} \ln \frac{n}{s}\right)\right) \\
= & o(1),
\end{aligned}
$$

where the last equality follows from $p \geq 32(\ln n) / n$ and $s \leq n / 100$.

We now state a lower bound on $\min -\operatorname{seed}(n, p)$ that is independent of $p$.

Corollary 8. Let $n$ be a positive integer and $p \in$ $[0,1]$. With probability $1-o(1)$,

$$
\min -\operatorname{seed}(n, p)=\Omega\left(n^{\frac{2}{3}}\right) .
$$

Proof. Invoke Theorem 4 for $p<n^{-2 / 3}$ and Theorem 7 otherwise.

Our proof of Theorem 7 actually shows the following stronger result.

Corollary 9. Let $n$ be a positive integer and $p \in$ $[32(\ln n) / n, 1]$. For a sufficiently small constant $\epsilon>$ 0 and any $s \leq \min \left\{n / 100, \epsilon p^{2} n^{2}\right\}$,

$$
\operatorname{Pr}\left[\exists S \subsetneq[n],|S|=s,|c(S) \backslash S| \geq \frac{p n}{8}\right]=o(1) .
$$

Building on Corollary 9, we obtain the following lower bound on the minimum number of seeds needed to convince a $\delta \in(0,1]$ fraction of vertices in $G(n, p)$.

Corollary 10. Let $n$ be a positive integer, $p \in$ $[32(\ln n) / n, 1]$ and $\delta \in(0,1]$. With probability $1-o(1)$ over the random graphs $G(n, p)$, at least $\Omega\left(\min \left\{\delta n, p^{2} n^{2}\right\}\right)$ seeds are needed to convince a $\delta$ fraction of the vertices. 
Proof. In the proof, all probabilities are taken over the random graphs $G(n, p)$. First, suppose $99 \delta n / 100 \geq p n / 8$. For a sufficiently small constant $\epsilon>0$,

$$
\begin{aligned}
& \begin{array}{l}
\operatorname{Pr}\left[\exists S \subsetneq[n],|S|=\min \left\{\frac{\delta n}{100}, \epsilon p^{2} n^{2}\right\},\right. \\
\operatorname{Pr}\left[\exists S \subsetneq[n],|S|=\min \left\{\frac{\delta n}{100}, \epsilon p^{2} n^{2}\right\},\right.
\end{array} \\
& \left.|c(S) \backslash S| \geq \frac{99 \delta n}{100}\right] \\
& \leq \operatorname{Pr}\left[\exists S \subsetneq[n],|S|=\min \left\{\frac{\delta n}{100}, \epsilon p^{2} n^{2}\right\},\right. \\
& \left.|c(S) \backslash S| \geq \frac{p n}{8}\right] \\
& =o(1) \text {, } \\
& \operatorname{Pr}[\exists S \subsetneq[n],|S|<\delta n,|c(S)| \geq \delta n] \\
& \leq \operatorname{Pr}[\exists S \subsetneq[n],|S|<\delta n, S \subsetneq c(S)] \\
& \leq \operatorname{Pr}[\exists S \subsetneq[n],|S|<\delta n, \exists v \in[n],|N(v)|<2|S|] \\
& \leq \operatorname{Pr}[\exists v \in[n],|N(v)|<2 \delta n] \\
& \leq \operatorname{Pr}\left[\exists v \in[n],|N(v)|<\frac{p n}{2}\right] \\
& \leq \sum_{v \in[n]} \operatorname{Pr}\left[|N(v)|<\frac{p n}{2}\right] \\
& =o(1) \text {, }
\end{aligned}
$$

where the last equation follows from Eq. (4) in the proof of Theorem 7 .

\section{Random seeds}

In this section, we extend the proof of Theorem 7 to deal with random seeds.

Theorem 11. Let $n$ be a positive integer and $p \in$ $[0,1]$. Let $S$ be picked uniformly at random from the family of all $n /(d \ln n)$-sized subsets of $[n]$ and consider the spreading of a message in $G(n, p)$ beginning from $S$. If $d>0$ is a sufficiently large constant, then with probability $1-o(1), c(S) \neq[n]$.

Sketch of proof. We shall mimic the proof of Theorem 7. In the sequel, all probabilities are taken over both $G(n, p)$ and $S$. Also, we set $s=n /(d \ln n)$. For $p \geq 32(\ln n) / n$ and any sufficiently large constant $d$,

$$
\begin{aligned}
& \operatorname{Pr}[c(S)=[n]] \\
\leq & \operatorname{Pr}\left[|c(S) \backslash S| \geq \frac{p n}{8}\right] \\
\leq & \operatorname{Pr}\left[\exists v \in[n],|N(v)|<\frac{p n}{2}\right] \\
& +\operatorname{Pr}\left[\left(|c(S) \backslash S| \geq \frac{p n}{8}\right) \wedge\left(\forall v \in[n],|N(v)| \geq \frac{p n}{2}\right)\right] \\
\leq & \sum_{v \in[n]} \operatorname{Pr}\left[|N(v)|<\frac{p n}{2}\right] \\
& +\operatorname{Pr}\left[\left(|c(S) \backslash S| \geq \frac{p n}{8}\right) \wedge\left(\forall v \in[n],|N(v)| \geq \frac{p n}{2}\right)\right] \\
\leq & \sum_{v \in[n]} \operatorname{Pr}\left[|N(v)|<\frac{p n}{2}\right]+\left(\begin{array}{c}
n-s \\
\frac{p n}{8}
\end{array}\right) \exp \left(-\frac{p^{2} n^{2}}{128} \ln \frac{n}{8 s}\right) \\
\leq & n \exp \left(-\frac{p n}{9}\right)+\left(\begin{array}{c}
n-s \\
\frac{p n}{8}
\end{array}\right) \exp \left(-\frac{p^{2} n^{2}}{128} \ln \frac{n}{8 s}\right) \\
\leq & n \exp \left(-\frac{p n}{9}\right)+n^{\frac{p n}{8}} \exp \left(-\frac{p^{2} n^{2}}{128}\right) \\
= & o(1) .
\end{aligned}
$$

Above, Eq. (10) follows from Lemma 6, which can be easily shown to hold for random seed sets; Eq (11) follows from Eq. (4) in the proof of Theorem 7; the last two inequalities are straightforward calculations.

Next, we turn to the case of $0<p<32(\ln n) / n$. For any sufficiently large constant $d$, Theorem $4 \mathrm{im}$ plies that with probability $1-o(1)$, at least

$$
\Omega\left(\min \left\{n, \frac{1}{p}\right\}\right)=\Omega\left(\frac{n}{\ln n}\right)>\frac{n}{d \ln n}
$$

seeds, randomly chosen or otherwise, are needed to convince all vertices.

A slight modification to Theorem 11 yields tighter bounds for the case of $p>32(\ln n) / n$. In fact, Eqs. (6)-(13) in Theorem 11 hold even if $S$ is picked uniformly at random from the family of all $n / 100-$ sized subsets of $[n]$ and $s=n / 100$, provided $p>$ $32(\ln n) / n$. In other words, in slightly dense (i.e., $p>32(\ln n) / n)$ random graphs, an $\Omega(n)$ number of random seeds are needed if we want an $\Omega(1)$ probability of convincing all vertices. This is summarized in the following corollary.

Corollary 12. Let $n$ be a positive integer and $p \in$ $[0,1]$. Let $S$ be picked uniformly at random from the family of all $n / 100$-sized subsets of $[n]$ and consider the spreading of a message in $G(n, p)$ beginning from $S$. If $p>32(\ln n) / n$, then $c(S) \neq[n]$ holds with probability $1-o(1)$.

Note that $n / 100$ is an asymptotically optimal bound as $n$ seeds trivially suffice to convince all vertices.

As a final remark, Theorem 11 and Corollary 12 still hold if $S$ consists of $n /(d \ln n)$ and $n / 100$, respectively, independent and uniformly random samples from $[n]$, with repetitions removed. This is because if $S$ is formed by picking $s \in \mathbb{N}$ independent and uniformly random samples from $[n]$, then by adding to $S$ a uniformly chosen $(s-|S|)$-sized set (from all $(s-|S|)$-sized subsets of $[n] \backslash S)$, the resulting $S$ distributes as if it has been chosen uniformly at random from the family of all $s$-sized subsets of $[n]$.

\section{Conclusion}

Our lower bounds on min-seed $(n, p)$ limit the convincing of a message to vertices in $G(n, p)$ beginning from 
any small (i.e., $\left.o\left(n^{2 / 3}\right)\right)$ set of seeds. Clearly, the limitations are still valid even if the choices of seeds can be made dynamically during the spreading process. We also give a much tighter bound of $\Omega(n / \ln n)$ on the number of randomly chosen seeds needed to convince all vertices. It is, however, future work to close the gap between the $\Omega\left(n^{2 / 3}\right)$ lower bound and the trivial $O(n)$ upper bound on min-seed $(n, p)$. Similarly, it remains to close the gap between the $\Omega(n / \ln n)$ lower bound for random seeds and the trivial $O(n)$ upper bound. It is also future work to generalize the lower bounds to more realistic settings where, for example, the belief in a message is represented by a real number rather than the binary full or none. Finally, the introduction of mutual trust and distrust between pairs of individuals, as proposed by Guha et. al. [Guha et al., 2004], may have influences on the convincing of a message to individuals and may be worth further research.

\section{References}

Artz, D. and Gil, Y. [2007], 'A survey of trust in computer science and the semantic web', Journal of Web Semantics: Science, Services and Agents on the World Wide Web 5(2), 58-71.

Bollobás, B. [2001], Random Graphs, 2nd edn, Cambridge University Press.

Chang, C. L. and Lyuu, Y. D. [2008], Spreading messages, in $\mathrm{X}$. Hu and J. Wang, eds, 'Proceedings of the 14th Annual International Computing and Combinatorics Conference', Springer-Verlag Berlin Heidelberg, pp. 587-599.

Chernoff, H. [1952], 'A measure of the asymptotic efficiency of tests of a hypothesis based on the sum of observations', Annals of Mathematical Statistics 23, 493-507.

Doerr, B., Friedrich, T. and Sauerwald, T. [2008], Quasirandom rumor spreading, in 'Proceedings of the 19th annual ACM-SIAM symposium on Discrete algorithms', pp. 773-781.

Gleeson, J. P. and Cahalane, D. J. [2007], 'Seed size strongly affects cascades on random networks', Physical Review E 75(056103).

Grandison, T. and Sloman, M. [2000], 'A survey of trust in internet applications', IEEE Communications Surveys and Tutorials 3(4), 2-16.

Guha, R., Kumar, R., Raghavan, P. and Tomkins, A. [2004], Propagation of trust and distrust, in 'Proceedings of the 13th International World Wide Web Conference', pp. 403-412.

Haykin, S. [1998], Neural Networks: A Comprehensive Foundation, 2nd edn, Prentice Hall.

Hedetniemi, S. T., Hedetniemi, S. M. and Liestman, A. L. [1988], 'A survey of broadcasting and gossiping in communication networks', Networks 18, 319 349.

Hromkovic, J., Klasing, R., Monien, B. and Peine, R. [1996], Dissemination of information in interconnection networks (broadcasting and gossiping), in D.-Z. Du and D. F. Hsu, eds, 'Combinatorial Network Theory', Kluwer Academic Publishers, pp. 125-212.

Karp, R., Schindelhauer, C., Shenker, S. and Vocking, B. [2000], Randomized rumor spreading, in 'Proceedings of the 41st IEEE Symposium on Foundations of Computer Science', pp. 565-574.
Motwani, R. and Raghaven, P. [1995], Randomized Algorithms, Cambridge University Press.

Schelling, T. C. [1973], 'Hockey helmets, concealed weapons, and daylight saving: A study of binary choices with externalities', Journal of Conflict Resolution 17(3), 381-428.

Watts, D. J. [2002], 'A simple model of global cascades on random networks', Proceedings of the $\mathrm{Na}$ tional Academy of Science 99(9), 5766-5771. 
